\title{
Crush cytology: an expeditious diagnostic tool for gastrointestinal tract malignancy
}

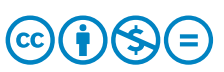

\author{
Authors \\ Ritesh Prajapati ${ }^{2}$, Nisharg Patel ${ }^{2}$, Krishna Parekh ${ }^{4}$

\section{Institutions} \\ 1 Department of Endoscopy \& Endosonography, Surat \\ Institute of Digestive Sciences (SIDS), Surat, India \\ 2 Department of Gastroenterology, Surat Institute of \\ Digestive Sciences (SIDS), Surat, India \\ 3 Department of Pathology, Surat Institute of Digestive \\ Sciences (SIDS), Surat, India \\ 4 Department of Clinical Research, Surat Institute of \\ Digestive Sciences (SIDS), Surat, India
}

Pankaj Desai ${ }^{1}$, Mayank Kabrawala ${ }^{2}$, Chintan Patel ${ }^{1}$, Priya Arora ${ }^{3}$, Rajiv Mehta ${ }^{2}$, Subhash Nandwani ${ }^{2}$, Parika Kalra ${ }^{2}$,

submitted 10.6 .2020

accepted after revision 30.12 .2020

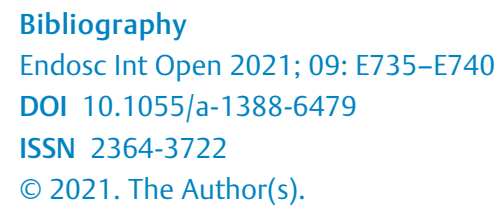

This is an open access article published by Thieme under the terms of the Creative Commons Attribution-NonDerivative-NonCommercial License, permitting copying and reproduction so long as the original work is given appropriate credit. Contents may not be used for commercial purposes, or adapted, remixed, transformed or built upon. (https://creativecommons.org/licenses/by-nc-nd/4.0/)

Georg Thieme Verlag KG, Rüdigerstraße 14,

70469 Stuttgart, Germany

Corresponding author

Pankaj Desai, MS, FASGE, FAIGE, Department of Endoscopy \& Endosonography, Surat Institute of Digestive Sciences (SIDS), Majura Gate, Ring Road, Surat, India-395002, India

Fax: +919824100681

drp_desai@hotmail.com

\section{ABSTRACT}

Background and study aims Crush cytology is a simple and rapid method used for diagnosis of central nervous system lesions. We have evaluated the diagnostic accuracy of crush cytology for gastrointestinal tract lesions.

Patients and methods This was a prospective, cross-sectional, single center study, conducted on the patients who had suspected malignant lesions between August 2018 and March 2020. The crush cytologic diagnoses were correlated with histology to determine the diagnostic accuracy.

Results During the period of interest, a total of 451 patients (26.4\% esophagus \& GE junction, $16.6 \%$ stomach, $5.9 \%$ ampulla \& duodenum, and $50.9 \%$ colorectal) had a suspected malignant lesion on endoscopic examination. Histology confirmed $92.9 \%$ cases as malignant lesions and $7.1 \%$ as nonmalignant. On crush cytology, $84.5 \%$ were positive for malignancy, $8.9 \%$ were negative for malignancy and $6.6 \%$ were reported as suspicious for malignancy. The overall sensitivity, specificity, positive predictive value, negative predictive value, and diagnostic accuracy of crush cytology were 97.3\%, 90\%, 99.2\%, 72.5\% and 96.9\%, respectively.

Conclusions Crush cytology is a highly sensitive, specific, rapid and cost effective technique to diagnose gastrointestinal malignancies in endoscopically suspected malignant lesions. However, it cannot entirely substitute histopathological examination for definite tumor typing, grading, confirming invasion and in cases in which cytology is suspicious. Crush cytology is an added asset to the histology to maximize diagnostic accuracy and accelerating decision making for the management of lesions.

\section{Introduction}

As a group, gastrointestinal cancers are not only one of the most common cancers but also one of the most common causes of cancer related mortality. According to the GLOBOCAN 2018 data, colorectal malignancy is the third and gastric cancer is the fifth most common cancer worldwide, and colorectal cancer is the second and gastric cancer the third leading cause of cancer related mortality. The global cancer burden is estima- ted to have risen to 18.1 million new cases and 9.6 million deaths in 2018, according to GLOBOCAN 2018 data. In India the estimated incidence of cancer is 89.4 per 100,000 and cancer-related mortality is 61.4 per 100,000 population [1]. In India, the incidence of gastric and colorectal cancer ranks sixth and seventh among most common cancers. Worldwide, the most commonly diagnosed gastrointestinal cancers include colorectal, gastric, liver (e.g. hepatocellular carcinoma), esophageal, and pancreatic. The advent of flexible fibreoptic video 
endoscopy with narrow band imaging (NBI) and endoscopic ultrasonography has greatly enhanced the ability to closely visualize several part of the gastrointestinal tract and obtain specimens under direct vision for histologic evaluation[2]. It is well accepted that the ultimate diagnosis of malignancy is based on histopathologic evaluation (HPE). The utility of cytology in the diagnosis of luminal gastrointestinal tract tumors is not addressed adequately in comparison to the HPE. Cytological evaluation can be done using brush cytology or crush smear technique. The pooled sensitivity and specificity of brush cytology for diagnosis of gastrointestinal malignancy has been reported as $83.4 \%$ and $80.9 \%$, respectively. The efficacy of all the cytological techniques is still continuously being assessed $[3,4]$.

Crush cytology is utilized as an excellent adjunct tool for central nervous system lesions, especially for intraoperative diagnosis [5]. Crush cytology is simple, easy to perform, cost effective and gives rapid results with minimal resources which could be an added asset to the histopathology [5]. Its efficacy and utility in guiding extent of neurosurgical resection has been proven, but there are a few studies in the literature on application of crush cytology for gastrointestinal malignancy $[3,6]$. Thus, the present study was undertaken to look at the reliability of crush cytology against the gold standard histopathology of gastrointestinal lesions.

\section{Patients and methods}

\section{Patients}

This was a prospective, cross-sectional, single-center study. Patients were enrolled between August 2018 and March 2020. A total of 11,690 endoscopic procedures were carried during this period and all patients who underwent either gastroscopy, colonoscopy, or side viewing scopy and were suspected to have malignant lesions on endoscopy were included in the study. Endoscopy was performed using a standard Gastroscope (Olympus EVIS EXERAIII HQ190), Colonoscope (Olympus EVIS EXERAIII CF-H190 L) or Side viewing scope (Olympus EVIS EXERAlll TGF-Q180) depending on the site of the lesion. On endoscopy, the patients with visible mucosal lesions such as ulcerative growth, stricture, or polypoidal mass lesions were assessed and biopsy specimen was taken from suspicious lesions using a biopsy forcep (with central spike). First, biopsy tissue was used for preparation of crush smears and smears were fixed in methanol and rest of the four biopsy samples were collected for HPE in $10 \%$ buffered formalin. Then, the samples were sent to the laboratory, where the crush smears were processed for cytology immediately and biopsy tissue was processed and sections cut from paraffin embedded blocks and stained with hematoxylin and eosin (H\&E) for evaluation. Biopsy slides were available for evaluation usually after 24 hours. The final HPE report was available within 4 days.

\section{Preparation of crush smears}

After taking biopsy, the endoscopist prepared the slide and sent it to the laboratory for cytofixation. For crush smears, a single biopsy tissue was gently crushed (squashed) between the two glass slides. A slide used for crushing tissue was kept at right angle to the slide on which tissue was placed. This slide was immediately fixed in methanol. It was then transported to the laboratory and then stained with H\&E. Staining time for crush cytology smear was about 45 minutes. The reporting time for the crush cytology smear was about one hour from the time of taking endoscopic biopsy. The crush smears and histopathological sections were examined by single well-versed pathologist who was blinded for the name, demographics and crush cytology findings but had an access to the endoscopic findings. All the findings were checked twice and final consensus diagnosis report was evaluated. The crush cytology diagnosis was recorded under three categories: positive for malignancy, suspicious of malignancy and negative for malignancy. A positive diagnosis on crush was given when there were unequivocal malignant cell clusters with good cellularity on smears. Smears which revealed low cellularity, or which showed only few atypical clusters, which were quantitatively or qualitatively insufficient to make a confident diagnosis of malignancy were reported as suspicious of malignancy. Negative for malignancy was reported in cases with definite absence of malignant or atypical cells or features consistent with inflammatory lesion. The results of crush cytology were compared with the histopathology results and histopathology was considered as the gold standard. For statistical purposes, suspicious category was considered as positive for malignancy.

\section{Statistical analysis}

Sensitivity, specificity, accuracy, positive predictive value and negative predictive value were calculated by comparison of HPE reports. Comparison and significance between crush cytology and histopathologic diagnosis, using chi-square test, were calculated with the SPSS statistical package, version 16.0 (SPSS Inc., Chicago, Illinois, United States).

\section{Results}

During the period of interest, a total of 451 patients were included with suspicious malignant lesions on endoscopic examination. The lesions were detected in the esophagus and gastroesophageal junction (26.4\%), stomach (16.6\%), ampulla and duodenum (5.9\%), and colon and rectum (50.9\%). There were $64 \%$ men with a mean age of $59.2 \pm 15.7$ years. Of 451 lesions, histology confirmed 419 cases of malignant lesions and 32 cases of nonmalignant lesions ( $>$ Table 1 ). The nonmalignant lesions included three tuberculosis, two amoebic colitis/ ameboma, 12 inflammatory lesion/ulcers, six adenomatous lesions with low-grade dysplasia, four high-grade dysplasia, one infarcted necrotic lipoma, one hyperplastic polyp, one inflammatory cap polyposis, and two serrated adenomas.

On crush cytology, 381 were diagnosed as positive for malignancy, 30 were suspicious for malignancy, and 40 were negative for malignancy. The cytomorphologic diagnosis is illustrated in $>$ Table 2 . The correlation of crush cytology and histopathological diagnosis of malignant lesions for the gastrointestinal tract are shown in $>$ Table 3 ( $P=0.860 ; \mathrm{X} 2$ test). The sensitivity and specificity of crush cytology for gastrointestinal tract malignancies were $97.3 \%$ and $90 \%$. The observed positive predictive value was $99.2 \%$ and negative predictive value was 
- Table 1 Crush cytological diagnoses of the study population.

\begin{tabular}{|l|c|c|c|c|}
\hline Biopsy site & Diagnostic Category & & \\
\hline & Positive for malignancy & Suspicious for malignancy & Negative for malignancy \\
\hline Esophagus + GEJ & 113 & 3 & 3 & 119 \\
\hline Gastric & 64 & 6 & 5 & 75 \\
\hline Colorectal & 183 & 18 & 3 & 29 \\
\hline Ampullary+duodenal & 21 & 3 & 40 & 27 \\
\hline Total & 381 & 30 & & 451 \\
\hline GEJ, gastroesophageal junction. & & & \\
\hline
\end{tabular}

- Table2 Cytohistologic diagnosis of study population (crush cytology and histopathology).

\begin{tabular}{|c|c|c|c|c|c|}
\hline Biopsy site & $\begin{array}{l}\text { Malignancy } \\
\text { on crush and } \\
\text { histology (n) }\end{array}$ & $\begin{array}{l}\text { Malignancy on crush } \\
\text { and nonmalignant } \\
\text { on histology ( } n \text { ) }\end{array}$ & $\begin{array}{l}\text { Nonmalignant on } \\
\text { crush and malignant } \\
\text { on histology ( } n \text { ) }\end{array}$ & $\begin{array}{l}\text { Nonmalignant on } \\
\text { crush and histology } \\
\text { (n) }\end{array}$ & $\begin{array}{l}\text { Total } \\
\text { (n) }\end{array}$ \\
\hline Esophagus + GEJ & 116 & 0 & 0 & 3 & 119 \\
\hline Gastric & 70 & 0 & 0 & 5 & 75 \\
\hline Colorectal & 200 & 1 & 10 & 19 & 230 \\
\hline Ampullary+duodenum & 22 & 2 & 1 & 2 & 27 \\
\hline Total (n) & 408 & 3 & 11 & 29 & 451 \\
\hline
\end{tabular}

- Table 3 Cytomorphologic categorization of gastrointeestinal malignancy using crush cytology.

\begin{tabular}{|c|c|c|c|c|c|}
\hline \multirow[t]{2}{*}{ Cytomorphology } & \multicolumn{4}{|l|}{ Site } & \multirow[t]{2}{*}{ Total } \\
\hline & Esophagus + GEJ (n) & Gastric (n) & Colorectal (n) & Ampullary + duodenum (n) & \\
\hline Squamous cell carcinoma & 85 & 0 & 2 & 0 & 87 \\
\hline Adenocarcinoma & 17 & 50 & 172 & 18 & 257 \\
\hline Poorly differentiated carcinoma & 8 & 9 & 6 & 4 & 27 \\
\hline Non-Hodgkin lymphoma & 2 & 2 & 1 & 0 & 5 \\
\hline Neuroendocrine tumor & 0 & 1 & 1 & 0 & 2 \\
\hline Spindle cell neoplasm/GIST & 0 & 2 & 1 & 0 & 3 \\
\hline Total & 113 & 64 & 183 & 22 & 381 \\
\hline
\end{tabular}

72.5\%. In addition, the crush cytology achieved $100 \%$ accuracy for the esophagus, gastroesophageal junction and gastric lesions.

In esophagus, well to moderately differentiated squamous cell carcinomas could be diagnosed as squamous type ( Fig.1a) with confidence but in moderately poor to poorly differentiated cases were difficult to diagnose the exact type on crush cytology alone. Crush cytology smears from small ampullary and periampullary lesions were most difficult to diagnose, particularly small polyps, small proliferative lesions, because these lesions had low cellularity, well differentiated na- ture, and lack of architectural details. Such a lesion showed presence of few atypical clusters which could represent regenerative atypia. The lesions with grossly malignant appearing large masses on endoscopy did not impose much diagnostic problem. Moreover, the adenocarcinomas ( $>$ Fig.1b) could be diagnosed successfully on cytology alone and majority of cases were observed in colorectal lesions. But for poorly differentiated carcinoma, non-Hodgkin lymphoma (NHL) (> Fig.1c), neuroendocrine tumor (NET) ( $>$ Fig.1d), spindle cell neoplasms ( $>$ Fig.1e) biopsy and immunohistochemistry confirmation was required. Cases of lymphoma could be diagnosed as malignant 


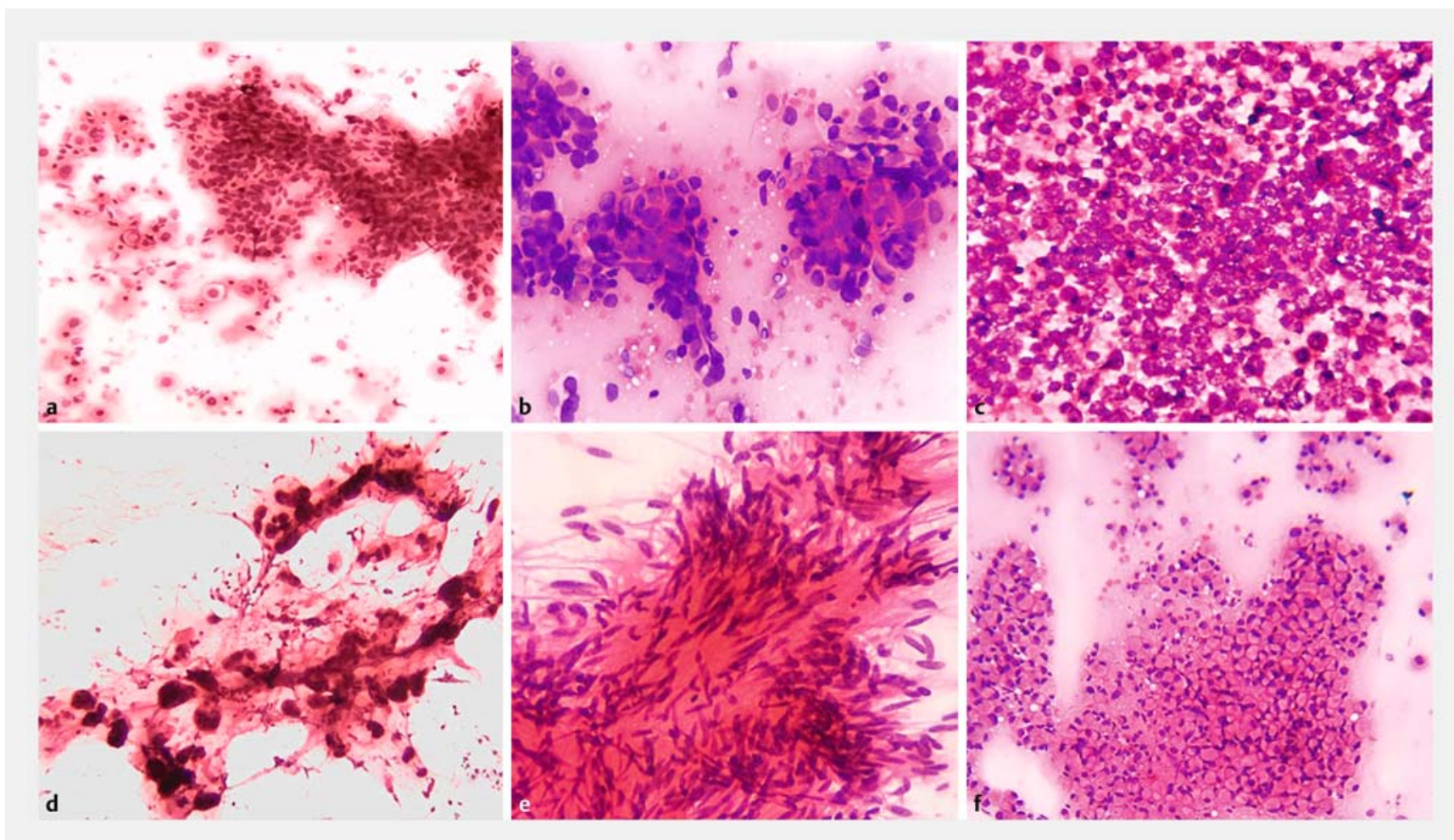

Fig. 1 Crush cytology. a Squamous cell carcinoma. b Adenocarcinoma. c Non-Hodgkin lymphoma. d Mucin-secreting adenocarcinoma. e Spindle cell neoplasm. $\mathbf{f}$ Signet ring carcinoma.

round cell tumors which revealed a possibility of NHL that could be confirmed by HPE and immunohistochemistry. It was observed that cases with either in situ/intramucosal lesion revealed only focal high-grade dysplasia (>Fig.1f) on crush due to low cellularity. Whereas, in invasive malignancies the smears were highly cellular with majority of cell clusters showing unequivocal features of malignancy like nuclear enlargement, pleomorphism, hyperchromasia, marked anisonucleosis, crowding, overlapping, loss of polarity, abnormal mitoses.

\section{Discussion}

Determining the true nature of gastrointesttinal tract lesions is crucial. Early diagnosis of gastrointestinal tract lesions with endoscopic evaluation plays a decisive role for their management. Depending upon the location and type of lesions various cytological techniques may be employed for primary diagnosis of lesions. Numerous studies have highlighted the diagnostic pitfalls of brush and touch smear cytology [3, 4, 7]. However, there are a few reported studies in the literature on the role of crush cytology in diagnosis of gastrointestinal tract malignancy. Crush cytology is a simple technique and can be performed with the available equipment in endoscopy and pathology laboratory [6]. In the present study, we have evaluated the diagnostic efficacy of crush cytology for malignancy of the gastrointestinal tract. We assessed sensitivity of $97.3 \%$, specificity of $90 \%$ and overall diagnostic accuracy of $96.9 \%$.
Batra et al. [3] and Chaithra et al. [6] proposed that crush cytology and histopathology have equivalent diagnostic reliability for malignancy of large intestine, stomach and esophagus. In the present study, we have evaluated the efficacy of crush smears for whole gastrointestinal tract. Batra et al. [3] reported sensitivity of crush smears $89.7 \%$ for gastroesophageal lesions. In the same way, Chaithra et al. [6] compared crush cytology with histopathology of resected specimens of large intestine and reported the sensitivity of $96 \%$ and specificity of $63.2 \%$. In our study the sensitivity and specificity of crush cytology for esophageal, GE junction and gastric lesions was 100\%, while the sensitivity and specificity was $95.2 \%$ and $95 \%$ respectively for colorectal lesions ( $\triangleright$ Table 4 ).

Cytology can be conducted by many ways; brush, crush and touch imprints are just a few names. Brush cytology is indeed an advantageous procedure as it provides wide area to investigate. Usually, it takes longer time, which is uncomfortable for the patients. Likewise, touch imprints are unpredictable, in cases of tumor with deeper infiltration. In consequence of these major pitfalls we might land up with inadequate diagnosis. Apart from the consequences of cytological procedures, it requires additional efforts and equipment's to yield the diagnosis. Whereas crush cytology requires minute amount of tissue, no additional equipment and efforts are needed and these together make crush cytology cost-effective. Most importantly, crush cytology produces rapid diagnosis within an hour in contrast to histopathological examination, which takes 3 days. However, histopathological examination cannot be replaced 
- Table 4 Diagnostic correlation between crush cytology and histopathology for gastrointestinal malignancy.

\begin{tabular}{|l|c|c|c|c|}
\hline Site & Sensitivity (\%) & Specificity & PPV & NPV \\
\hline Gastrointestinal tract & 97.3 & 90.0 & 99.2 & 72.5 \\
\hline Esophageal+GEJ & 100 & 100 & 100 & 100 \\
\hline Gastric & 100 & 100 & 100 & 100 \\
\hline Colorectal & 95.2 & 95.0 & 99.5 & 65.5 \\
\hline Ampullary+ Duodenal & 95.6 & 50.0 & 91.6 & 66.6 \\
\hline GEJ, gastroesophageal junction; PPV, positive predictive value; NPV, negative predictive value & & \\
\hline
\end{tabular}

completely by crush cytology for tumor typing, grading, and confirmation of invasion.

It would be expected to have a suspicious finding on crush cytology. Even with histologic examination, a few cases are inconclusive because of suspicious findings. The inference is that the smear is low cellularity or there was a sampling error. In the present study, 30 cases of crush cytology with suspicion of malignancy but not definitive were identified and considered positive for statistical analysis only, not for surgical decision making. In cases with suspicious but not conclusive findings, the clinician has always been advised to wait for the histopathology report. Statistics applied without including these cases had sensitivity, specificity, positive predictive value, and negative predictive values of $97.1 \%, 96.7 \%, 99.7 \%$, and $72.5 \%$ respectively.

Crush artifacts in squash cytology were not a major problem. In fact, cell morphology was well preserved in almost all cases and acceptable for interpretation, except in an occasional case of necrotic mass lesion. Crush cytology smears from very-welldifferentiated tumors showed bland nuclear features on cytology. But those with low cellularity from markedly ulcerated and necrotic lesions or from stricturing lesions showed few atypical clusters that could not be confidently diagnosed as malignant on crush alone and were the reason of false negatives and an occasional false-positive result. Thus, well-differentiated tumors or tumors with low-grade morphology pose a major problem in the diagnosis using crush cytology, thus resulting in a low negative predictive value. In the present study, 11 cases of malignancy were negative on crush but positive on histology. Among them, 10 cases were colorectal malignancies. The possible explaination would be as follows: Six cases were well-differentiated adenocarcinoma with low-grade morphology, which was difficult to diagnose on crush cytology. Three were low-grade lymphoma, which were difficult to distinguish from inflammatory lesions without referring architectural features. One case was a NET, which was predominantly submucosal and had lymphovascular tumor emboli, so it was not seen in the crush smears. In all such cases, "wait for biopsy policy" should be followed. Well-differentiated adenocarcinoma could be diagnosed on histology, wherein architecture and invasion can be assessed. It is emphasized that in cases of negative or suspicious results on cytology, the biopsy report should always be awaited before decisions are made about radical surgical management, unless there is a surgical emergency. We suggest that biopsy should always be performed in formalin for histopathological examination along with crush to avoid unnecessary repeat endoscopy if crush cytology is negative or suspicious. Moreover, if crush cytology is positive, further management can be immediately planned and we may not have to wait for a biopsy report. Lymphoma, gastrointestinal stromal tumor, and NET showed features that suggest the diagnosis in some cases but definitely require confirmation of biopsy and immunohistochemistry. In these cases, we can only suggest a diagnosis, which needs to be confirmed on histology. In the present study, crush cytology diagnosed NHL, three gastrointestinal stromal tumors, and two NETs. Low-grade lymphoma with bland cytology was difficult to diagnose on cytology and resulted in three false negatives. One case of NET was also false negative. On the other hand, poorly differentiated NETs cannot be accurately differentiated by cytology and are difficult to differentiate from adenocarcinoma, even on histopathologic examination.

Immunohistochemistry, therefore, is needed in these cases. In addition, NBI is another diagnostic modality to distinguish malignant lesions on endoscopic examination. However, NBI provided subjective findings which may have inter-observer variability. In contrast to that crush cytology provided objective findings to diagnose malignancy. Hence, further study is required to correlate findings of crush cytology with NBI.

\section{Conclusions}

In conclusion, crush cytology appears to be a highly sensitive, specific, rapid and cost effective procedure to diagnose gastrointestinal malignancies in endoscopically suspected malignant lesions. Moreover, crush cytology for lesions in the esophagus, gastroesophageal junction, and stomach is most reliable. Cytomorphologic evaluation on crush cytology along with good correlation of clinical and endoscopic findings is quick enough to deliver the diagnosis on the same day of endoscopy, which saves time and accelerates the decision making for the management of malignant lesion.

\section{Competing interests}

The authors declare that they have no conflict of interest. 


\section{References}

[1] Bray F, Ferlay J, Soerjomatarm I et al. Global Cancer Statistics 2018: GLOBOCAN estimates of incidence and mortality worldwide for 36 cancers in 185 countries. Ca Cancer J Clin 2018; 68: 394-424

[2] Barbeiro S, Libanio D, Castro R et al. Narrow-band imaging: clinical application in gastrointestinal endoscopy. GE Port J Gastroenterol 2018; 26: 40-53

[3] Batra M, Handa U, Sachdev A. Comparison of cytohistologic techniques in diagnosis of gastroesophageal malignacy. Acta Cytologica 2006; 52: 77-82

[4] Kaur S, Sharma R, Kaushal V et al. Diagnostic accuracy of endoscopic brush cytology in malignancies of upper gastrointestinal tract: A pro- spective study of 251 patients in North India. J Cancer Res Ther 2016; 12: 681-684

[5] Yadav KK, Bhatti R, Moorchung $N$ et al. Is crush cytology of central nervous system lesions relevant in surgical practice today? J Neurosci Rural Pract 2019; 10: 34-38

[6] Gv C, Saha D, Yadav R et al. The Role of crush cytology in the diagnosis of large-intestine lesions with correlation on histopathology. Acta Cytol 2018; 62: 215-222

[7] Adhya AK, Mohanty R. Utility of touch imprint cytology in the preoperative diagnosis of malignancy in low resource setting. Diagn Cytopathol 2017; 45: 507-512 